\title{
Editorial
}

\section{Through Neutrino Eyes: The Search for New Physics}

\author{
Abhijit Samanta, ${ }^{1}$ David Latimer, ${ }^{2}$ and Michael A. Schmidt ${ }^{3}$ \\ ${ }^{1}$ Department of Physics, Heritage Institute of Technology, Kolkata 700 107, India \\ ${ }^{2}$ Department of Physics, University of Puget Sound, Tacoma, WA, USA \\ ${ }^{3}$ School of Physics A28, University of Sydney, Sydney, NSW 2006, Australia \\ Correspondence should be addressed to Abhijit Samanta; abhijit.samanta@gmail.com
}

Received 28 October 2014; Accepted 28 October 2014

Copyright (C) 2015 Abhijit Samanta et al. This is an open access article distributed under the Creative Commons Attribution License, which permits unrestricted use, distribution, and reproduction in any medium, provided the original work is properly cited. The publication of this article was funded by $\mathrm{SCOAP}^{3}$.

The year 2014 will mark the 60th anniversary since the neutrino detector of Frederick Reines and Clyde L. Cowan, Jr. was turned (neutrino detection in 1956). After many years, Super-Kamiokande [1] showed in 1998 that neutrinos are massive. Today, neutrino physics has become a very active research field: there is a plethora of different neutrino experiments and theoretical studies. Subsequent measurements [2-6] of the two neutrino mass squared differences and the leptonic mixing parameters lead to a phase of precision experiments in neutrino physics. Recently the last remaining mixing angle, the 1-3 mixing angle, has been measured by the Daya Bay [7, 8], Double Chooz [9, 10], and RENO [11] experiments after initial hints by T2K [12] and MINOS $[13,14]$. Contrary to theoretical expectations from flavor symmetry considerations, it turned out to be large.

The next main goals of the experimental program are the measurement of the mass hierarchy and the Dirac CP phase, which is facilitated by the relatively large 1-3 mixing angles. These measurements will help to pin down the theoretical origin of neutrino mass and mixing, for example, confirming or refuting the idea of a flavor symmetry in the lepton sector. Additionally precision measurements of neutrino properties will also permit using neutrinos as a tool for probing new physics connected with neutrinos, like dark matter or dark energy. There are a huge number of ongoing and upcoming neutrino experiments worldwide studying these issues.

To celebrate the 60th anniversary of the first neutrino detector, we have collected original research articles as well as review articles for this special issue focusing mainly on physics at underground detectors and its complementary studies at the LHC to uncover the nature of neutrinos as well as physics beyond standard model.
The article "The low-scale approach to neutrino masses" by S. M. Boucenna et al. provides a short review on lowscale models of neutrino mass generation including the phenomenological potential signatures associated with direct neutrino mass messenger production at the LHC, messengerinduced lepton flavor violation processes, and the presence of WIMP cold dark matter candidates.

The article "Beyond standard model searches in the MiniBooNE experiment" by Teppei Katori and Janet Conrad provides a review on the contribution of the MiniBooNE Experiment to beyond standard model searches in the neutrino sector. MiniBooNE observed excesses of $v_{e}$ and anti$v_{e}$ candidate events in neutrino and antineutrino mode, respectively. To date, these excesses have not been explained within the neutrino Standard Model, the Standard Model extended by three massive neutrinos. The results set for the first time strict limits on Lorentz violating extensions of the Standard Model. Most recently, MiniBooNE is running with a beam tuned in beam-dump mode to search for dark sector particles.

The article "Searching for neutrinoless double-beta decay of ${ }^{130}$ Te with CUORE" by D. R. Artusa et al. provides a review on the experimental techniques used in Cryogenic Underground Observatory for Rare Events (CUORE), its current status, and anticipated physics reach. CUORE is an upcoming experiment designed to search for neutrinoless double-beta decay of ${ }^{130} \mathrm{Te}$ using an array of $988 \mathrm{TeO}_{2}$ crystal bolometers operated at $10 \mathrm{mK}$. The detector will contain $206 \mathrm{~kg}$ of ${ }^{130} \mathrm{Te}$ and have an average energy resolution of $5 \mathrm{keV}$; the projected half-life sensitivity after five years of live time is $1.610^{26} \mathrm{y}$ at $1 \sigma\left(9.5 \times 10^{25} \mathrm{y}\right.$ at the $90 \%$ confidence 
level), which corresponds to an upper limit on the effective Majorana mass in the range 40-100 meV (50-130 meV).

The article "Neutrinos as probes of Lorentz invariance" by J. S. Díaz reviews generic experimental signatures of the breakdown of Lorentz symmetry in the neutrino sector. Neutrino oscillations are identified as an ideal experimental setup to search for breaking of Lorentz symmetry.

The article "Constraints on light neutrino parameters derived from the study of neutrinoless double beta decay" by S. Stoica and A. Neacsu revises the present constraints on the neutrino mass parameters derived from the decay analysis of the experimentally interesting nuclei using the latest results for the phase space factors and nuclear matrix elements (NMEs), as well as for the experimental lifetime limits. This restricts the range of spread of the NME values calculated with different methods and, hence, to reduce the uncertainty in deriving limits for the Majorana neutrino mass parameter. These results may be useful to have an updated image on the present neutrino mass sensitivities associated with measurements for different isotopes and to better estimate the range of values of the neutrino masses that can be explored in the future double beta decay experiments.

The article "Theory of neutrino-atom collisions: the history, present status, and BSM physics" by K. A. Kouzakov and Alexander I. Studenikin provides an overview of the current theoretical studies on neutrino-atom scattering processes. The ionization channel of these processes, which is studied in experiments searching for neutrino magnetic moments, is discussed. Recent developments in the theory of atomic ionization by the impact of reactor antineutrinos are also enlightened here.

The article "Entanglement in a QFT model of neutrino oscillations" by M. Blasone et al. uses the tools of quantum information theory to provide a convenient description of the phenomena of particle mixing and flavor oscillations in terms of entanglement using a suitable entanglement measure, the concurrence, that allows extracting the two-mode (flavor) entanglement from full multimode, multiparticle flavor neutrino states.

The article "Gaseous detector with sub-keV threshold to study neutrino scattering at low recoil energies" by A. V. Kopylov et al. discusses the construction of a gaseous detector with a sub-keV electron equivalent threshold, which allows a precision measurement of the neutrino magnetic moment and to observe coherent scattering of neutrinos on nuclei. The progress in the development of low noise electronics makes it possible to register rare events at the threshold less than $100 \mathrm{eV}$.

The article "Performance of water-based liquid scintillator: an independent analysis" by $\mathrm{D}$. Beznosko et al. discusses a water-based liquid scintillator, which is based on the idea of dissolving an organic scintillator in water using special surfactants. The results show that a goal of 100 optical photons/MeV has been achieved. Simulations have indicated that this is an optimal light yield for observing both the Cerenkov ring and the scintillation light from proton decay in a large water detector.
The papers included in this special issue cover a small number of the diverse issues in neutrino physics. We are certain that the short reviews on different aspects in neutrino physics will help new researchers and the original research articles will have an impact on the future development of neutrino physics.

\section{Abhijit Samanta \\ David Latimer \\ Michael A. Schmidt}

\section{References}

[1] Y. Fukuda, T. Hayakawa, E. Ichihara et al., "Evidence for oscillation of atmospheric neutrinos," Physical Review Letters, vol. 81, no. 8, pp. 1562-1567, 1998.

[2] M. Apollonio, A. Baldini, C. Bemporad et al., "Limits on neutrino oscillations from the $\mathrm{CHOOZ}$ experiment," Physics Letters B, vol. 466, pp. 415-430, 1999.

[3] Q. Ahmad, R. C. Allen, T. C. Andersen et al., "Direct evidence for neutrino flavor transformation from neutral-current interactions in the Sudbury Neutrino Observatory," Physical Review Letters, vol. 89, Article ID 011301, 2002.

[4] S. N. Ahmed, A. E. Anthony, E. W. Beier et al., "Measurement of the total active ${ }^{8} \mathrm{~B}$ solar neutrino flux at the sudbury neutrino observatory with enhanced neutral current sensitivity," Physical Review Letters, vol. 92, Article ID 181301, 2004.

[5] K. Eguchi, S. Enomoto, K. Furuno et al., "First results from KamLAND: evidence for reactor antineutrino disappearance," Physical Review Letters, vol. 90, Article ID 021802, 2003.

[6] M. Ahn, E. Aliu, S. Andringa et al., "Measurement of neutrino oscillation by the K2K experiment," Physical Review D, vol. 74, no. 7, Article ID 072003, 39 pages, 2006.

[7] F. An, J. Z. Bai, A. B. Balantekin et al., "Observation of electronantineutrino disappearance at daya bay," Physical Review Letters, vol. 108, Article ID 171803, 2012.

[8] F. P. An, Q. An, J. Z. Bai et al., "Improved measurement of electron antineutrino disappearance at Daya Bay," Chinese Physics C, vol. 37, no. 1, Article ID 011001, 2013.

[9] Y. Abe, C. Aberle, T. Akiri et al., "Indication of reactor $\bar{\nu}_{e}$ disappearance in the double chooz experiment," Physical Review Letters, vol. 108, no. 13, Article ID 131801, 7 pages, 2012.

[10] Y. Abe, C. Aberle, J. C. dos Anjos et al., "Reactor $\bar{V}_{e}$ disappearance in the Double Chooz experiment," Physical Review D, Article ID 052008, 2012.

[11] J. Ahn, S. Chebotaryov, J. H. Choi et al., "Observation of reactor electron antineutrinos disappearance in the RENO experiment," Physical Review Letters, vol. 108, no. 19, 6 pages, 2012.

[12] K. Abe, N. Abgrall, Y. Ajima et al., "Indication of electron neutrino appearance from an accelerator-produced off-axis muon neutrino beam," Physical Review Letters, vol. 107, Article ID 041801, 2011.

[13] D. G. Michael, P. Adamson, T. Alexopoulos et al., "Observation of muon neutrino disappearance with the MINOS detectors in the NuMI neutrino beam," Physical Review Letters, vol. 97, Article ID 191801, 2006.

[14] P. Adamson, D. J. Auty, D. S. Ayres et al., "Improved search for Muon-neutrino to electron-neutrino oscillations in MINOS," Physical Review Letters, vol. 107, no. 18, Article ID 181802, 6 pages, 2011. 

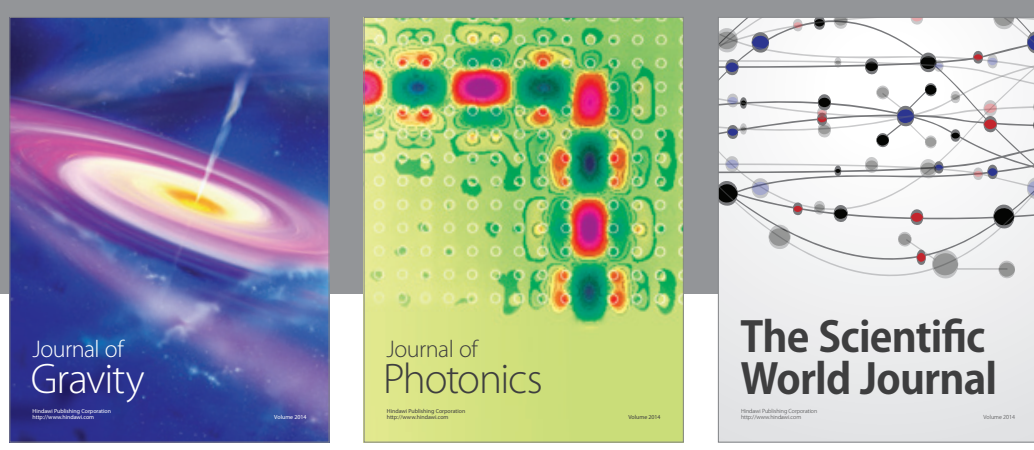

The Scientific World Journal
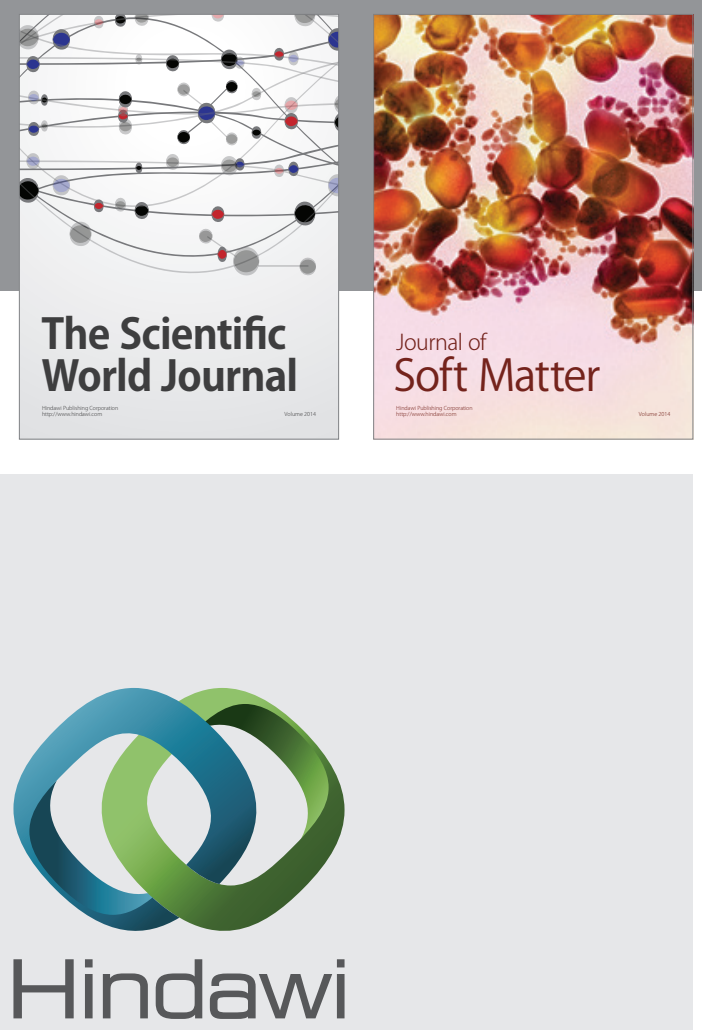

Submit your manuscripts at

http://www.hindawi.com

nternational Journal of

Statistical Mechanics
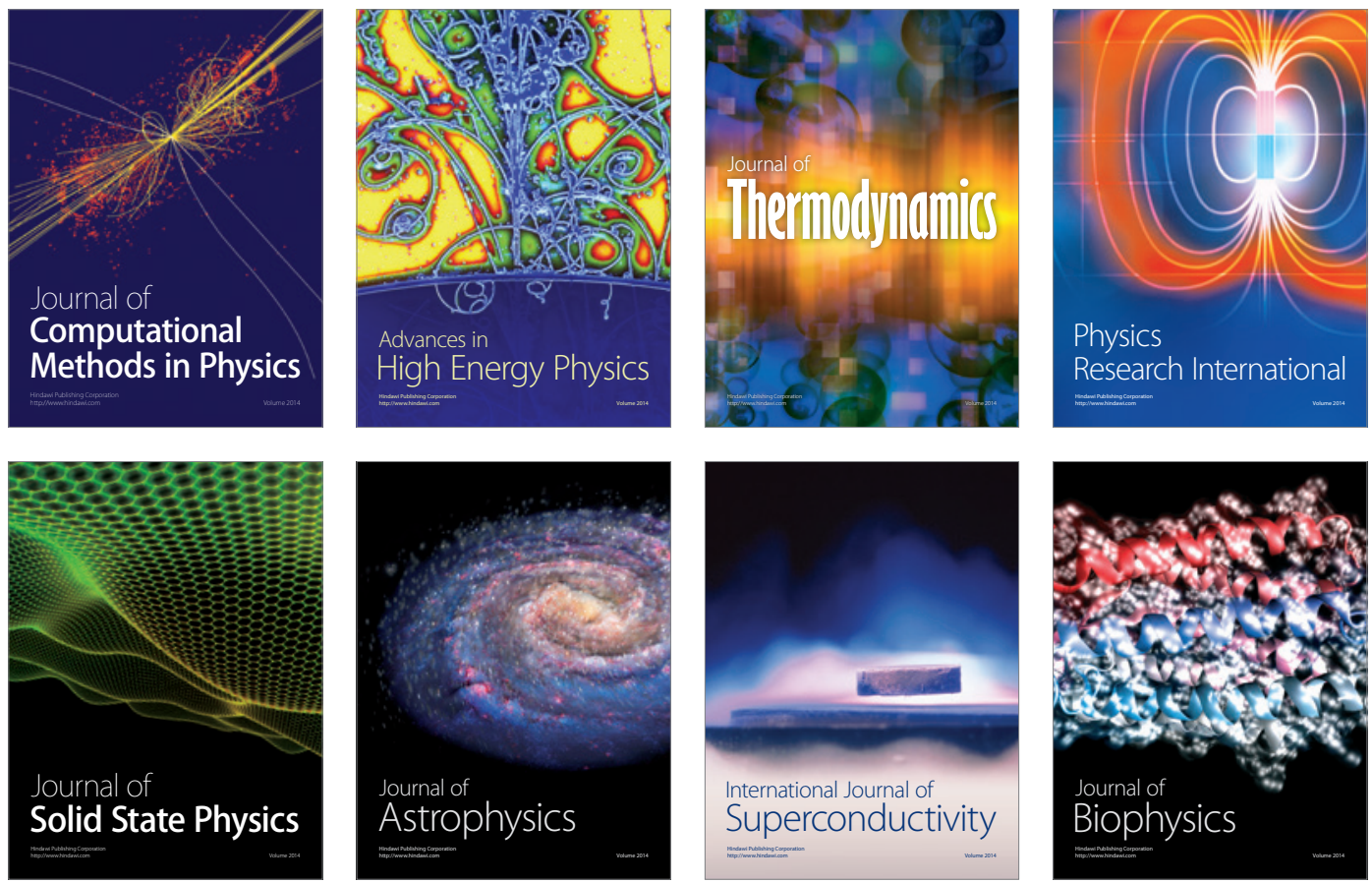
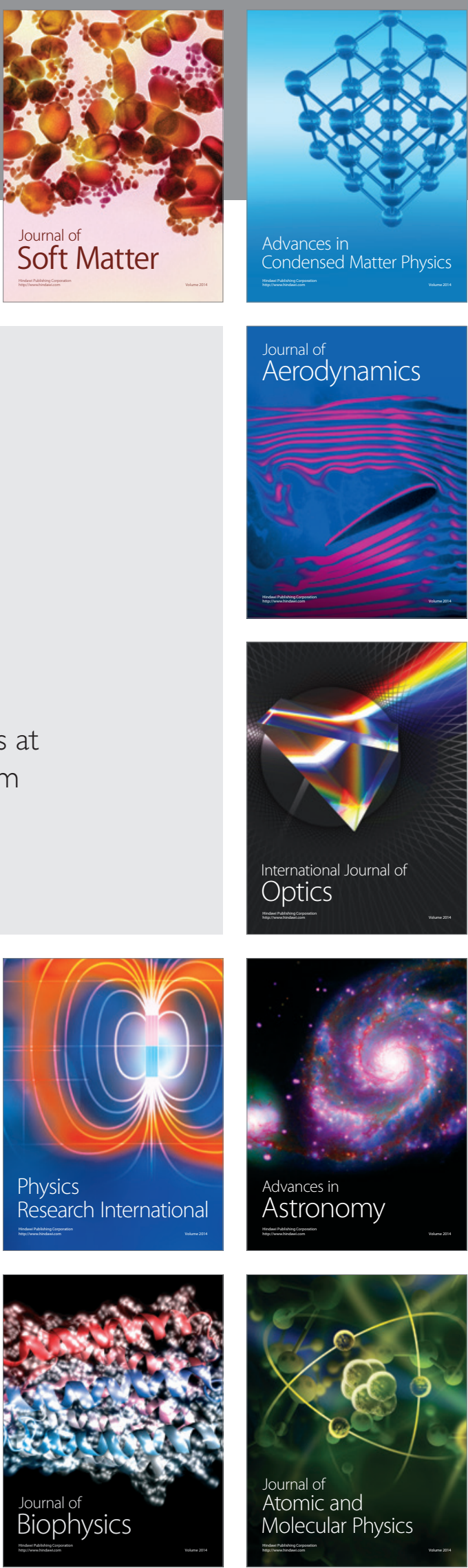